\title{
MINIMAL EFFORT PROBLEMS AND THEIR TREATMENT BY SEMISMOOTH NEWTON METHODS*
}

\author{
KAZUFUMI $\mathrm{ITO}^{\dagger}$ AND KARL KUNISCH ${ }^{\ddagger}$
}

\begin{abstract}
The paper introduces minimum effort control problems. These provide an answer to the question of the smallest possible control bound which still allows us to drive the system to a target within a fixed time $T$. This is a counterpart to the time optimal control problem which minimizes the time required to drive the system to the target, given a control bound. The problem is formulated as an optimal control problem with pointwise constraint on the control. The necessary conditions of optimality are derived by Lagrange multiplier theory. The semismooth Newton method is applied to a properly regularized problem. Well-posedness and superlinear convergence of the semismooth Newton method are proved for linear control systems under a controllability condition. Numerical results are presented for demonstrating the applicability and feasibility of the proposed method.
\end{abstract}

Key words. minimum effort control, Lagrange multiplier theory, semismooth Newton method

AMS subject classifications. 49K15, 49M15, 65K10

DOI. $10.1137 / 100784667$

1. Introduction. The objective of this paper is to introduce and investigate minimal effort problems which are formulated as

$$
\left\{\begin{array}{cl}
\min & \frac{1}{q} \sum_{j=1}^{m}\left|u_{j}\right|_{L^{\infty}(0, T)}^{q} \\
\text { subject to } & \frac{d}{d t} x(t)=f(x(t), u(t)) \text { on }(0, T), \\
& x(0)=x_{0}, \quad g(x(T))=0
\end{array}\right.
$$

where $q \geq 1$ and $T$ are fixed. Here $x$ and $u$ denote the state and the control variable of the dynamical system that arises as a constraint in (1.1), with the conditions on $f$ to be specified below. The mapping $g$ characterizes the terminal constraint on the state. It is assumed that at least one admissible control $u$ exists for which the constraints in (1.1) are met and such that the cost is finite. Solving (1.1) requires us to find that control $u$ for which the magnitude of each control-coordinate is as small as possible uniformly over the time horizon $(0, T)$. Subsequently the resulting $q$-mean over the $L^{\infty}(0, T)$ norms of the coordinates is minimized.

${ }^{*}$ Received by the editors February 1, 2010; accepted for publication (in revised form) June 29, 2011; published electronically October 6, 2011.

http://www.siam.org/journals/sicon/49-5/78466.html

$\dagger$ Department of Mathematics, North Carolina State University, Raleigh, NC 27695-8205 (kito@ math.ncsu.edu). This author's research was partially supported by the Army Research Office grants 56349-MA and 49308-MA, and Air Force Research Office grant FA9550-09-1-0520.

${ }^{\ddagger}$ Institut für Mathematik, Karl-Franzens-Universität Graz, A-8010 Graz, Austria (karl.kunisch@ uni-graz.at). This author's research was supported in part by the Fonds zur Förderung der wissenschaftlichen Forschung under SFB 32, "Mathematical Optimization and Applications in Biomedical Sciences." 
It can readily be observed that this problem is equivalent to

$$
\left\{\begin{array}{cl}
\min & \frac{1}{q} \sum_{j=1}^{m}\left(\gamma_{j}\right)^{q} \\
\text { subject to } & \frac{d}{d t} x(t)=f(x(t), \gamma u(t)) \text { on }(0, T), \\
& x(0)=x_{0}, g(x(T))=0, \quad u(t) \in[-1,1]^{m}, \quad \gamma \in\left(\mathbb{R}^{+}\right)^{m} .
\end{array}\right.
$$

This is an optimal control problem with bilateral constraints on the control $u$, and product structure with respect to the variables $\gamma, u$. The notation $\gamma u$ is used for pointwise vector multiplication, i.e., $(\gamma u)_{i}=\gamma_{i} u_{i}$ for $i=1, \ldots, m$ and $\gamma, u \in \mathbb{R}^{m}$. Further, $[-1,1]^{m}$ denotes the $m$-dimensional cube with center zero and axis-parallel edges of length $2, \mathbb{R}^{+}=[0, \infty)$, and $\left(\mathbb{R}^{+}\right)^{m}=\otimes_{i=1}^{m} \mathbb{R}^{+}$. From the point of view of numerical realization the pointwise bounds on the controls constitute a significant obstacle, since the controls can be expected to be of bang-bang type. For this purpose semismooth Newton methods, combined with suitable regularization techniques, can be very efficient. We refer to [IK1, IK2, IK3, U] and the references given there. It should be noted that the proposed approach does note require that the controls be bang-bang, and in particular, no a priori knowledge of the structure of the optimal control is used for the algorithm.

The minimum effort problem has received relatively little attention in the literature. However, let us quote $[\mathrm{N}]$, where sufficient conditions are given for the optimal control to be bang-bang for several classes of minimum effort problems. In [BCB] multiple shooting and parametric techniques to solve minimum effort problems for linear systems are presented. We also quote $[\mathrm{BPW}]$ for a more recent survey of solving control problems with bang-bang structure. For time-optimal control problems, a popular numerical approach depends on reparametrization of the original problem, for example, in terms of switching times or arc durations; see, e.g., [MB, KN]. These techniques typically require a priori information, e.g., on the number of switching points, which is generally not available for (1.1). Such a type of information is not required for the algorithm that we propose.

In a more recent paper [BMT], the authors compare indirect methods, involving the shooting method and a homotopy technique, to a direct method based on an interior point formulation, on a problem involving bang-bang controls and singular arcs. Second order sufficient optimality conditions for state constraint bang-bang optimal control problems and time optimal control problems with box constraints on the controls are investigated in [MAG, MBKK] and solved by a direct numerical method based on sequential quadratic programming, which simultaneously checks the fulfilment of the second order conditions. In [VM] optimal control problems with $L^{1}$ cost of the controls are considered. A precise analysis of the bang-bang nature of the controls is made and second order sufficient optimality conditions are given.

The paper is organized as follows. Section 2 contains the precise problem formulation and a well-posedness result. The optimality system for (1.1) is derived in section 3. It is shown that the optimal control may be of the bang-bang type. Since a semismooth Newton method is not directly applicable to this system, we introduce a regularized problem in section 4 . The convergence of the optimal control of the regularized problem is established. The regularized problem has an advantage that the optimality condition provides the complete synthesis and the optimal control is Lipschitz continuous. Section 5 contains the analysis of the semismooth Newton method for the case of linear systems. Well-posedness and superlinear convergence are proved under a controllability condition. Numerical results are given in the final section 6 . 
2. Problem statement and basics. We consider for $q \geq 1$ and $x_{0} \in \mathbb{R}^{n}$

$$
\left\{\begin{array}{cl}
\min & \frac{1}{q} \sum_{j=1}^{m}\left(\gamma_{j}\right)^{q} \\
\text { subject to } & \frac{d}{d t} x(t)=f(x(t), \gamma u(t)) \text { on }(0, T), \\
& x(0)=x_{0}, \quad g(x(T))=0, \\
& u \in U_{a d}, \quad \gamma \in\left(\mathbb{R}^{+}\right)^{m} .
\end{array}\right.
$$

Here $\gamma u \in \mathbb{R}^{m}$ is defined by $(\gamma u)_{j}=\gamma_{j} u_{j}, 1 \leq j \leq m$, and

$$
U_{a d}=\left\{u \in L^{1}\left(0, T ; \mathbb{R}^{m}\right): u(t) \in U=[-1,1]^{m} \text {, a.e. } t \in(0, T)\right\} .
$$

Further $g \in C^{1}\left(\mathbb{R}^{n}, \mathbb{R}^{r}\right)$ and $f \in C^{1}\left(\mathbb{R}^{n} \times \mathbb{R}^{m}, \mathbb{R}^{n}\right)$, and it satisfies, for continuous, nondecreasing functions $c_{0}, c_{1}, c_{2}: \mathbb{R}^{+} \rightarrow \mathbb{R}^{+}$, and any $x \in \mathbb{R}^{n}, v \in \mathbb{R}^{m}, v_{1} \in \mathbb{R}^{m}$, $v_{2} \in \mathbb{R}^{m}$,

$$
\begin{gathered}
|f(x, v)|+\left|\frac{\partial}{\partial x} f(x, v)\right| \leq c_{0}(|x|)+c_{1}(|x|)|v|, \\
\left|f\left(x, v_{1}\right)-f\left(x, v_{2}\right)\right| \leq c_{2}(|x|)\left|v_{1}-v_{2}\right|, \\
(f(x, v), x)_{\mathbb{R}^{n}} \leq\left(a_{1}+a_{2}|v|\right)\left(1+|x|^{2}\right) \text { for some } a_{1}, a_{2} \in \mathbb{R}^{+},
\end{gathered}
$$

where $(\cdot, \cdot)_{\mathbb{R}^{n}}$ denotes the Euclidean inner product in $\mathbb{R}^{n}$. It can be checked that $(2.3)$ is satisfied, for example, provided that (2.1) and (2.2) hold with $c_{0}$ and $c_{2}$ bounded from above by affine functions and $\frac{\partial}{\partial x} f(x, 0)$ is globally bounded with respect to $x$.

We shall further use the following condition:

For every $x \in W^{1, \infty}\left(0, T ; \mathbb{R}^{n}\right)$ and every sequence $\left\{v_{k}\right\}_{k=1}^{\infty}$ converging weakly in $L^{2}\left(0, T ; \mathbb{R}^{m}\right)$ to some $v \in L^{2}\left(0, T ; \mathbb{R}^{m}\right)$, there exists a subsequence $\left\{v_{k_{\ell}}\right\}_{\ell=1}^{\infty}$ such that $\lim f\left(x, v_{k_{\ell}}\right)=f(x, v)$ weakly in $L^{2}\left(0, T ; \mathbb{R}^{n}\right)$.

Note that (2.4) is satisfied, for instance, for $f(x, v)=f_{1}(x)+B(x) v$, if, for $x \in W^{1, \infty}\left(0, T ; \mathbb{R}^{n}\right)$, we have $B(x) \in L^{\infty}\left(0, T ; \mathbb{R}^{n \times m}\right.$, and $\left.f_{1} \in C\left(\mathbb{R}^{n}, \mathbb{R}^{n}\right)\right)$.

Exploiting the pointwise bounds on the controls we have the following result.

Proposition 2.1. Let (2.1)-(2.3) hold. Then for every $x_{0} \in \mathbb{R}^{n}, v \in U_{\text {ad }}$ there exists a unique solution $x=x\left(\cdot ; x_{0}, v\right) \in W^{1, \infty}\left(0, T ; \mathbb{R}^{n}\right)$ to

$$
\frac{d}{d t} x(t)=f(x(t), v(t)) \text { on }(0, T), x(0)=x_{0},
$$

and the mapping $\left(x_{0}, v\right) \rightarrow x$ is Lipschitz continuous from $\mathbb{R}^{n} \times L^{p}\left(0, T ; \mathbb{R}^{m}\right)$ to $W^{1, p}\left(0, T ; \mathbb{R}^{n}\right)$, uniformly on bounded sets in $\mathbb{R}^{n} \times L^{p}\left(0, T ; \mathbb{R}^{m}\right), 1 \leq p \leq \infty$.

Proof. Let $x_{0} \in \mathbb{R}^{n}, v \in U$. Then by (2.1) and the standard theory of ordinary differential equations, there exists a unique solution $x \in W^{1,1}\left(0, \tau ; \mathbb{R}^{n}\right)$ for some $\tau \in$ $(0, T]$ to $(2.5)$. From $(2.3)$

$$
\frac{1}{2} \frac{d}{d t}|x(t)|^{2}=\left(\frac{d}{d t} x(t), x(t)\right)_{\mathbb{R}^{n}}=(f(x(t), v(t)), x(t))_{\mathbb{R}^{n}} \leq k(t)\left(1+|x(t)|^{2}\right),
$$

where $k(t)=a_{1}+a_{2}|v(t)|$, and thus for $t \in(0, \tau]$

$$
\begin{aligned}
|x(t)|^{2} & \leq \exp \left(2 \int_{0}^{t} k(s) d s\right)\left(\left|x_{0}\right|^{2}+2 \int_{0}^{t} k(s) d s\right) \\
& \leq \exp \left(2\left(T a_{1}+a_{2}|v|_{L^{1}}\right)\right)\left(\left|x_{0}\right|^{2}+2\left(T a_{1}+a_{2}|v|_{L^{1}}\right)\right)=: M .
\end{aligned}
$$

Copyright $@$ by SIAM. Unauthorized reproduction of this article is prohibited. 
The continuation method now implies the existence of a unique global solution to (2.5). Moreover, there exists a constant $C=C\left(|v|_{L^{1}}\right)$ such that

$$
|x|_{C\left([0, T], \mathbb{R}^{n}\right)} \leq C\left(\left|x_{0}\right|+|v|_{L^{1}}+1\right) .
$$

By (2.1) there exists a constant $\tilde{C}=\tilde{C}\left(\left|x_{0}\right|,|v|_{L^{\infty}}\right)$ such that $\left|x\left(x_{0}, v\right)\right|_{W^{1, \infty}}$ $\leq \tilde{C}\left(\left|x_{0}\right|,|v|_{L^{\infty}}\right)$. For two solutions $x_{i}$ corresponding to $\left(x_{0, i}, v_{i}\right), i=1,2$, we have

$$
\begin{aligned}
& \left|x_{1}(t)-x_{2}(t)\right| \leq\left|x_{0,1}-x_{0,2}\right|+\int_{0}^{t}\left|f\left(x_{1}(s), v_{1}(s)\right)-f\left(x_{2}(s), v_{2}(s)\right)\right| d s \\
& \leq\left|x_{0,1}-x_{0,2}\right|+\int_{0}^{t}\left[\omega(s)\left|x_{1}(s)-x_{2}(s)\right|+c_{2}(M)\left|v_{1}(s)-v_{2}(s)\right|\right] d s
\end{aligned}
$$

where $\omega(s)=c_{0}(M)+c_{1}(M)\left|v_{1}(s)\right|$. By Gronwall's inequality this implies that

$$
\left|x_{1}(t)-x_{2}(t)\right| \leq \exp \left(\int_{0}^{t} \omega(s) d s\right)\left(\left|x_{0,1}-x_{0,2}\right|+c_{2}(M) \int_{0}^{t}\left|v_{1}(s)-v_{2}(s)\right| d s,\right.
$$

and hence there exists $\hat{C}$ such that $\left|x_{1}-x_{2}\right|_{C\left([0, T], \mathbb{R}^{n}\right)} \leq \hat{C}(M)\left(\left|x_{0,1}-x_{0,2}\right|+\left|v_{1}-v_{2}\right|_{L^{1}}\right)$. Moreover $W^{1, p}\left(0, T ; \mathbb{R}^{n}\right)$ regularity of the solutions follows from (2.1), (2.2), and the fact that $v \in U_{a d}$.

Proposition 2.2. Assume that there exists at least one feasible triple $(x, v, \gamma)$ for $(\mathrm{P})$ and that (2.1)-(2.4) hold. Then there exists at least one optimal solution to $(\mathrm{P})$.

Proof. Let $\left\{\left(x_{k}, v_{k}, \gamma_{k}\right)\right\}_{k=1}^{\infty}$, with $x_{k}=x\left(v_{k}, \gamma_{k}\right)$, denote a minimizing sequence for (P). Then $\left\{\left(v_{k}, \gamma_{k}\right)\right\}_{k=1}^{\infty}$ is bounded in $L^{\infty}\left(0, T ; \mathbb{R}^{n}\right) \times \mathbb{R}^{m}$ and by Proposition 2.1 the sequence $\left\{x_{k}\right\}_{k=1}^{\infty}$ is bounded in $W^{1, \infty}\left(0, T ; \mathbb{R}^{n}\right)$. Hence there exists a subsequence, denoted by the same symbol, and $\left(x^{*}, v^{*}, \gamma^{*}\right) \in W^{1, \infty}\left(0, T ; \mathbb{R}^{n}\right) \times$ $L^{\infty}\left(0, T ; \mathbb{R}^{m}\right) \times \mathbb{R}^{m}$ such that $\left(x_{k}, v_{k}, \gamma_{k}\right) \rightarrow\left(x^{*}, v^{*}, \gamma^{*}\right)$ weakly in $W^{1,2}\left(0, T ; \mathbb{R}^{n}\right) \times$ $L^{2}\left(0, T ; \mathbb{R}^{m}\right) \times \mathbb{R}^{m}$. By $(2.1)$ and $(2.4)$ at $x=x^{*}$, we can pass to the limit, on a further subsequence, in $\frac{d}{d t} x_{k}=f\left(x_{k}, \gamma_{k} v_{k}\right)$ to obtain $\frac{d}{d t} x^{*}=f\left(x^{*}, \gamma^{*} v^{*}\right), x^{*}(0)=x_{0}$, and $g\left(x^{*}(T)\right)=0$. Hence $\left(x^{*}, v^{*}, \gamma^{*}\right)$ is feasible. Passing to the limit in $\sum_{j=1}^{m}\left(\gamma_{k}\right)_{j}^{q}$ we find that $\left(x^{*}, v^{*}, \gamma^{*}\right)$ is optimal for $(\mathrm{P})$.

3. Optimality system. This section is devoted to deriving an optimality system for $(\mathrm{P})$. Throughout the remainder of this paper it is assumed that (2.1)-(2.4) hold and that $\left(x^{*}, u^{*}, \gamma^{*}\right)$ is a solution to $(\mathrm{P})$. It is further required that $f$ be continuously Fréchet-differentiable as a mapping from $W^{1, \infty}\left(0, T ; \mathbb{R}^{n}\right) \times L^{\infty}\left(0, T ; \mathbb{R}^{m}\right)$ to $L^{\infty}\left(0, T ; \mathbb{R}^{n}\right)$ at $\left(x^{*}, \gamma^{*} u^{*}\right)$. For this to hold it suffices that the first derivative of $(x, v) \rightarrow f(x, v)$ is Lipschitz-continuous on bounded subsets of $\mathbb{R}^{n} \times \mathbb{R}^{m}$.

Let $(\mathrm{P})$ be expressed in the abstract form

$$
\min F(u, \gamma) \text { subject to } G(u, \gamma)=0 \text { over }(u, \gamma) \in U_{a d} \times\left(\mathbb{R}^{+}\right)^{m} \text {. }
$$

Here

$$
F(u, \gamma)=\frac{1}{q} \sum_{j=1}^{m}\left(\gamma_{j}\right)^{q}, \quad G(u, \gamma)=g(x(T)),
$$

where $x=x(u, \gamma)$, is the solution to the differential equation in $(\mathrm{P})$. The following regular point condition will be used:

$$
0 \in \operatorname{int}\left\{G_{u}\left(u^{*}, \gamma^{*}\right)\left(U_{a d}-u^{*}\right)+G_{\gamma}\left(u^{*}, \gamma^{*}\right)\left(\left(\mathbb{R}^{m}\right)^{+}-\gamma^{*}\right)\right\}
$$


see, e.g., [MZ, IK1]. With (3.2) and the regularity conditions on $f$ holding, there exists a Lagrange multiplier $\mu \in \mathbb{R}^{r}$ associated with the constraint $G(u, \gamma)=0$ such that the Lagrangian

$$
\mathcal{L}(u, \gamma, \mu)=F(u, \gamma)+(\mu, G(u, \gamma))_{\mathbb{R}^{r}}
$$

is stationary at $\left(u^{*}, \gamma^{*}\right)$ in the sense that

$$
\begin{gathered}
\mathcal{L}_{u}\left(u^{*}, \gamma^{*}, \mu\right)(u, \gamma)=\left(\mu, G_{u}\left(u^{*}, \gamma^{*}\right)\left(u-u^{*}\right)\right)_{\mathbb{R}^{r}} \geq 0 \text { for all } u \in U_{a d}, \\
\mathcal{L}_{\gamma}\left(u^{*}, \gamma^{*}, \mu\right)(u, \gamma)=F_{\gamma}\left(u^{*}, \gamma^{*}\right)+\mu^{T} G_{\gamma}\left(u^{*}, \gamma^{*}\right)=0 .
\end{gathered}
$$

Here and throughout the following we assume that

$$
\gamma_{j}^{*}>0 \text { for all } j=1, \ldots, r .
$$

From a practical point of view this is not a severe assumption and it avoids treating special cases or introducing additional Lagrange multipliers.

It is simple to argue that for $v \in L^{\infty}\left(0, T ; \mathbb{R}^{m}\right)$ we have

$$
G_{u}\left(u^{*}, \gamma^{*}\right)(v)=g^{\prime}\left(x^{*}(T)\right) h(T),
$$

where $h \in W^{1,2}\left(0, T ; \mathbb{R}^{n}\right)$ satisfies

$$
\frac{d}{d t} h=f_{x}\left(x^{*}, \gamma^{*} u^{*}\right) h+f_{u}\left(x^{*}, \gamma^{*} u^{*}\right)\left(\gamma^{*} v\right), \quad h(0)=0,
$$

and $f_{u}\left(x^{*}, \gamma^{*} u^{*}\right)$ stands for the Fréchet derivative of $f$ with respect to the second variable of $f$. Moreover, for $\delta \gamma \in \mathbb{R}^{m}$

$$
G_{\gamma}\left(u^{*}, \gamma^{*}\right)(\delta \gamma)=g^{\prime}\left(x^{*}(T)\right) \xi(T),
$$

where $\xi \in W^{1,2}\left(0, T ; \mathbb{R}^{n}\right)$ satisfies

$$
\frac{d}{d t} \xi=f_{x}\left(x^{*}, \gamma^{*} u^{*}\right) \xi+f_{u}\left(x^{*}, \gamma^{*} u^{*}\right)\left(u^{*} \delta \gamma\right), \quad \xi(0)=0 .
$$

Let us note that for the special case $g(x)=x-\bar{x}$, with $\bar{x} \in \mathbb{R}^{n}$ some fixed target, the regular point condition becomes a controllability-type condition with constraints on the controls: There exists some $\delta>0$ such that for each $r \in \mathbb{R}^{n}$ with $|r|_{\mathbb{R}^{n}}<\delta$ there exist $v \in U_{a d}$ and $\delta \gamma \geq 0$ such that

$$
r=h(T)+\xi(T),
$$

where $h$ is the solution to (3.7) with $v$ replaced by $v-u^{*}$, and $\xi$ is the solution to (3.9) with $\delta \gamma$ replaced by $\delta \gamma-\gamma^{*}$. Above $|\cdot| \mathbb{R}^{n}$ denotes the Euclidean norm in $\mathbb{R}^{n}$.

Let us specifically address the regular point condition (3.2) for linear control systems in the following lemma.

Lemma 3.1. For a linear control system with $f(x, u)=A x+B u$ with $A \in$ $\mathbb{R}^{n \times n}$ and $B \in \mathbb{R}^{n \times m}$, with target constraint given by $g(x)=x-\bar{x}, \bar{x} \in \mathbb{R}^{n}$ fixed, the regular point condition (3.2) holds, provided that $(A, B)$ is controllable, i.e., $\operatorname{rank}\left[B, A B, \ldots, A^{n-1} B\right]=n$. 
Proof. Since $g^{\prime}$ is the identity, we can use (3.7) and (3.9) to express (3.2) as the condition: There exists $\delta>0$ such that for every $r \in \mathbb{R}^{n}$ with $|r| \leq \delta$ there exist $v \in U_{a d}$ and $\delta \gamma \geq 0$ such that $r=z(T)=h(T)+\xi(T)$, where $z$ satisfies

$$
\left\{\begin{array}{l}
\frac{d}{d t} z(t)=A z(t)+B\left(\gamma^{*} v+\delta \gamma u^{*}-2 \gamma^{*} u^{*}\right), \\
z(0)=0
\end{array}\right.
$$

Choosing $\delta \gamma=2 \gamma^{*} \geq 0$ results in

$$
\left\{\begin{array}{l}
\frac{d}{d t} z(t)=A z(t)+B\left(\gamma^{*} v\right), \\
z(0)=0
\end{array}\right.
$$

Define $\Gamma=\operatorname{diag}\left(\gamma_{1}^{*}, \ldots, \gamma_{m}^{*}\right)$ and observe that $\Gamma$ is positive definite by the standing assumption (3.5). Hence the system $(A, B \Gamma)$ is controllable since $(A, B)$ is assumed to be controllable. It then follows that $0 \in \mathbb{R}^{n}$ is in the interior of the controllable set $\left\{z(T ; v): v \in U_{a d}\right\}$ [MS, page 30], as desired.

A first order necessary optimality condition is obtained next.

TheOREm 3.1. Let $\left(u^{*}, \gamma^{*}\right)$ be an optimal solution for (3.1) with associated state $x^{*}$. Assume that (3.5) and the regular point condition (3.2) are satisfied. Then there exist $(p, \mu) \in W^{1, \infty}\left(0, T ; \mathbb{R}^{n}\right) \times \mathbb{R}^{r}$ such that

$$
\left\{\begin{array}{l}
\frac{d}{d t} x^{*}=f\left(x^{*}, \gamma^{*} u^{*}\right), \quad x^{*}(0)=x_{0}, \quad g\left(x^{*}(T)\right)=0, \\
-\frac{d}{d t} p=f_{x}\left(x^{*}, \gamma^{*} u^{*}\right)^{T} p, \quad p(T)=g^{\prime}\left(x^{*}(T)\right)^{T} \mu, \\
\left(\gamma_{j}^{*}\right)^{q-1}+\int_{0}^{T} u_{j}^{*}\left(f_{u}\left(x^{*}, \gamma^{*} u^{*}\right)^{T} p\right)_{j} d t=0 \text { for each } j=1, \ldots, m, \\
\left(f_{u}\left(x^{*}(t), \gamma^{*} u^{*}(t)\right)^{T} p(t), u-u^{*}(t)\right) \geq 0 \text { for a.e. } t \in(0, T), \text { all } u \in U .
\end{array}\right.
$$

Proof. Let $\mu \in \mathbb{R}^{r}$ be as defined above, and let $p \in W^{1, \infty}\left(0, T ; \mathbb{R}^{n}\right)$ denote the solution to the adjoint equation. Further, $h$ is defined in (3.7). Then

$$
\begin{aligned}
\frac{d}{d t}(h(t), p(t))_{\mathbb{R}^{n}} & =\left(f_{x}(x, \gamma u) h+f_{u}(x, \gamma u)(\gamma v), p\right)_{\mathbb{R}^{n}}-\left(f_{x}(x, \gamma u) h, p\right)_{\mathbb{R}^{n}} \\
& =\left(f_{u}(x, \gamma u)(\gamma v), p\right)_{\mathbb{R}^{n}}
\end{aligned}
$$

where for simplicity we dropped the superscript * with $x, u$, and $\gamma$ and also suppressed their dependence on $t$ on the right-hand side equations. Similarly we find

$$
\begin{aligned}
\frac{d}{d t}(\xi(t), p(t))_{\mathbb{R}^{n}} & =\left(f_{x}(x, \gamma u) \xi+f_{u}(x, \gamma u) u \delta \gamma, p\right)_{\mathbb{R}^{n}}-\left(f_{x}(x, \gamma u) \xi, p\right)_{\mathbb{R}^{n}} \\
& =\left(f_{u}(x, \gamma u) u \delta \gamma, p\right)_{\mathbb{R}^{n}},
\end{aligned}
$$

where $\xi$ is given in (3.9). Integrating $(3.12)$ over $(0, T)$ we find

$$
(h(T), p(T))_{\mathbb{R}^{n}}=\left(g^{\prime}(x(T)) h(T), \mu\right)_{\mathbb{R}^{r}}=\int_{0}^{T}\left(f_{u}(x, \gamma u)(\gamma v), p\right)_{\mathbb{R}^{n}} d t,
$$

and hence by (3.6)

$$
\left(\mu, G_{u}(u, \gamma) v\right)_{\mathbb{R}^{r}}=\int_{0}^{T}\left(\gamma v, f_{u}(x, \gamma u)^{T} p\right)_{\mathbb{R}^{m}} d t .
$$

Copyright $@$ by SIAM. Unauthorized reproduction of this article is prohibited. 
With (3.3) this implies the last claim in (3.11). Similarly from (3.13)

$$
(\xi(T), p(T))_{\mathbb{R}^{n}}=\left(g^{\prime}(x(T)) \xi(T), \mu\right)_{\mathbb{R}^{r}}=\int_{0}^{T}\left(u \delta \gamma, f_{u}(x, \gamma u)^{T} p\right)_{\mathbb{R}^{m}} d t
$$

and thus by (3.8)

$$
\left(\mu, G_{\gamma}(u, \gamma) \delta \gamma\right)_{\mathbb{R}^{r}}=\int_{0}^{T}\left(u \delta \gamma, f_{u}(x, \gamma u)^{T} p\right)_{\mathbb{R}^{m}} d t .
$$

The third claim in the optimality system now follows from (3.4).

Remark 3.1. Concerning (3.5), the proof reveals that if $\gamma_{j}^{*}=0$ for some $j$, then $\int_{0}^{T} u_{j}^{*}\left(f_{u}\left(x^{*}, \gamma^{*} u^{*}\right)^{T} p\right)_{j} d t \leq 0$.

The last equation in (3.11) can equivalently be expressed as

$$
u_{j}^{*}(t) \in-\operatorname{sgn}\left(f_{u}\left(x^{*}(t), \gamma^{*} u^{*}(t)\right)^{T} p(t)\right)_{j}, \quad j=1, \ldots, m,
$$

where

$$
\operatorname{sgn}(s)=\left\{\begin{array}{ccc}
-1 & \text { if } & s<0 \\
{[-1,1]} & \text { if } & s=0 \\
1 & \text { if } & s>0
\end{array}\right.
$$

The next to last equation in (3.11) can therefore be expressed as

$$
\left(\gamma_{j}^{*}\right)^{q-1}-\int_{0}^{T}\left|\left(f_{u}\left(x^{*}(t), \gamma^{*} u^{*}(t)\right)^{T} p(t)\right)_{j}\right| d t=0 \quad \text { for } j=1, \ldots, m .
$$

For convenience we summarize the optimality system thus obtained:

$$
\left\{\begin{array}{l}
\frac{d}{d t} x^{*}=f\left(x^{*}, \gamma^{*} u^{*}\right), \quad x^{*}(0)=x_{0}, \quad g\left(x^{*}(T)\right)=0, \\
-\frac{d}{d t} p=f_{x}\left(x^{*}, \gamma^{*} u^{*}\right)^{T} p, \quad p(T)=g^{\prime}\left(x^{*}(T)\right)^{T} \mu, \\
\left(\gamma_{j}^{*}\right)^{q-1}-\int_{0}^{T}\left|\left(f_{u}\left(x^{*}(t), \gamma^{*} u^{*}(t)\right)^{T} p(t)\right)_{j}\right| d t=0 \quad \text { for } j=1, \ldots, m . \\
u_{j}^{*}(t) \in-\operatorname{sgn}\left(f_{u}\left(x^{*}(t), \gamma^{*} u^{*}(t)\right)^{T} p(t)\right)_{j}, \quad j=1, \ldots, m .
\end{array}\right.
$$

To solve (3.18) projection methods could be applied. Here, however, our interest lies in the development of higher order methods like Newton methods. For this purpose a regularization is introduced in the following section. As a salient feature we look for a regularization that only changes the system "as little as possible" so that a semismooth Newton method becomes applicable. In particular, we do not regularize sgn by a $C^{2}$ - or even $C^{\infty}$-function, like $-\frac{2}{\pi} \arctan (c x)$ for a large value of $c$.

4. Regularization. For $\varepsilon>0$ consider the family of regularized problems

$$
\left\{\begin{array}{cl}
\min \quad & \frac{1}{q} \sum_{j=1}^{m}\left(\gamma_{j}\right)^{q}+\frac{\varepsilon}{2} \int_{0}^{T} \sum_{j=1}^{m} \gamma_{j} u_{j}^{2}(t) d t \\
\text { subject to } & \frac{d}{d t} x(t)=f(x(t), \gamma u(t)) \text { on }(0, T) \\
& x(0)=x_{0}, \quad g(x(T))=0 \\
& u \in U_{a d}, \quad \gamma \in\left(\mathbb{R}^{+}\right)^{m}
\end{array}\right.
$$

Copyright $\odot$ by SIAM. Unauthorized reproduction of this article is prohibited. 
It is straightforward to argue that $\left(P_{\varepsilon}\right)$ admits a solution $\left(x^{\varepsilon}, u^{\varepsilon}, \gamma^{\varepsilon}\right)$ for each $\varepsilon>0$. In the following result, the asymptotic behavior as $\varepsilon \rightarrow 0$ is addressed. It will be convenient to introduce $|u|_{L_{\Gamma}^{2}}^{2}=\int_{0}^{T} \sum_{j=1}^{m} \gamma_{j} u_{j}^{2}(t) d t$.

Proposition 4.1. As $\varepsilon \rightarrow 0^{+}$the family $\left\{\left(x^{\varepsilon}, u^{\varepsilon}, \gamma^{\varepsilon}\right)\right\}_{\varepsilon>0}$ contains a subsequence that converges in $W^{1, p}\left(0, T ; \mathbb{R}^{n}\right) \times L^{p}\left(0, T ; \mathbb{R}^{m}\right) \times \mathbb{R}^{m}, p \in[1, \infty)$, to a solution of $(\mathrm{P})$. If the solution to $(\mathrm{P})$ is unique, then the whole family $\left\{\left(x^{\varepsilon}, u^{\varepsilon}, \gamma^{\varepsilon}\right)\right\}_{\varepsilon>0}$ converges to this solution.

Proof. Since the technique of this proof is standard we provide only the essential steps. Let $\left(\gamma^{*}, u^{*}\right)$ denote an optimal solution of $(\mathrm{P})$. Then for every $\varepsilon>0$

$$
\frac{1}{q} \sum_{j=1}^{m}\left(\gamma_{j}^{\varepsilon}\right)^{q}+\frac{\varepsilon}{2}\left|u^{\varepsilon}\right|_{L_{\Gamma^{\varepsilon}}^{2}}^{2} \leq \frac{1}{q} \sum_{j=1}^{m}\left(\gamma_{j}^{*}\right)^{q}+\frac{\varepsilon}{2}\left|u^{*}\right|_{L_{\Gamma^{*}}^{2}}^{2} .
$$

As a consequence $\left\{\gamma^{\varepsilon}\right\}_{\varepsilon>0}$ is bounded. Moreover, $\left\{\left(u^{\varepsilon}, x^{\varepsilon}\right)\right\}_{\varepsilon>0}$ is bounded in $L^{\infty}\left(0, T ; \mathbb{R}^{m}\right)$ $\times W^{1, \infty}\left(0, T ; \mathbb{R}^{n}\right)$ since $u^{\varepsilon} \in U_{a d}$ and by Proposition 2.1. Consequently there exist $(\bar{\gamma}, \bar{u}, \bar{x}) \in \mathbb{R}^{m} \times U_{a d} \times W^{1, \infty}\left(0, T ; \mathbb{R}^{n}\right)$ such that on a subsequence $\left(\gamma^{\varepsilon_{n}}, u^{\varepsilon_{n}}, x^{\varepsilon_{n}}\right) \rightarrow$ $(\bar{\gamma}, \bar{u}, \bar{x})$ weakly in $\mathbb{R}^{m} \times L^{2}\left(0, T ; \mathbb{R}^{m} \times W^{1,2}\left(0, T ; \mathbb{R}^{n}\right)\right)$. Using (2.4) at $x=\bar{x}$, we can pass to the limit, on a subsequence in $\frac{d}{d t} x^{\varepsilon_{n}}=f\left(x^{\varepsilon_{n}}, \gamma^{\varepsilon_{n}} u^{\varepsilon_{n}}\right)$, to obtain that $\frac{d}{d t} \bar{x}=f(\bar{x}, \bar{\gamma} \bar{u})$. Moreover, $\bar{x}(0)=x_{0}$ and $\bar{g}(\bar{x}(T))=0$. Passing to the limit in (4.1) we find that $(\bar{\gamma}, \bar{u})$ is a solution to $(\mathrm{P})$.

As a function of $\varepsilon$ the solutions to $\left(P_{\varepsilon}\right)$ satisfy the following monotonicity properties.

Proposition 4.2. Let $0<\varepsilon \leq \varepsilon^{\prime}$. Then

$$
\left|u^{\varepsilon^{\prime}}\right|_{L_{\Gamma^{\varepsilon^{\prime}}}^{2}} \leq\left|u^{\varepsilon}\right|_{L_{\Gamma^{\varepsilon}}^{2}} \leq\left|u^{*}\right|_{L_{\Gamma^{*}}^{2}}
$$

and

$$
\sum_{j=1}^{m}\left(\gamma_{j}^{*}\right)^{q} \leq \sum_{j=1}^{m}\left(\gamma_{j}^{\varepsilon}\right)^{q} \leq \sum_{j=1}^{m}\left(\gamma_{j}^{\varepsilon^{\prime}}\right)^{q} \leq \sum_{j=1}^{m}\left(\gamma_{j}^{*}\right)^{q}+\frac{q \varepsilon^{\prime} \sum_{j=1}^{m} \gamma_{j}^{*}}{2}
$$

for any solution $\left(u^{*}, \gamma^{*}\right)$ to $(\mathrm{P})$.

Proof. We have

$$
\frac{1}{q} \sum_{j=1}^{m}\left(\gamma_{j}^{\varepsilon}\right)^{q}+\frac{\varepsilon}{2}\left|u^{\varepsilon}\right|_{L_{\Gamma^{\varepsilon}}^{2}}^{2} \leq \frac{1}{q} \sum_{j=1}^{m}\left(\gamma_{j}^{\varepsilon^{\prime}}\right)^{q}+\frac{\varepsilon}{2}\left|u^{\varepsilon^{\prime}}\right|_{L_{\Gamma^{\varepsilon^{\prime}}}^{2}}^{2}
$$

and

$$
\frac{1}{q} \sum_{j=1}^{m}\left(\gamma_{j}^{\varepsilon^{\prime}}\right)^{q}+\frac{\varepsilon^{\prime}}{2}\left|u^{\varepsilon^{\prime}}\right|_{L_{\Gamma^{\varepsilon}}^{2}}^{2} \leq \frac{1}{q} \sum_{j=1}^{m}\left(\gamma_{j}^{\varepsilon}\right)^{q}+\frac{\varepsilon^{\prime}}{2}\left|u^{\varepsilon}\right|_{L_{\Gamma^{\varepsilon}}^{2}}^{2} .
$$

This implies that

$$
\frac{1}{q} \sum_{j=1}^{m}\left(\gamma_{j}^{\varepsilon}\right)^{q}+\frac{\varepsilon}{2}\left|u^{\varepsilon}\right|_{L_{\Gamma^{\varepsilon}}^{2}}^{2} \leq \frac{1}{q} \sum_{j=1}^{m}\left(\gamma_{j}^{\varepsilon}\right)^{q}+\frac{\varepsilon^{\prime}}{2}\left(\left|u^{\varepsilon}\right|_{L_{\Gamma^{\varepsilon^{\prime}}}^{2}}^{2}-\left|u^{\varepsilon^{\prime}}\right|_{L_{\Gamma^{\varepsilon}}^{2}}^{2}\right)+\frac{\varepsilon}{2}\left|u^{\varepsilon^{\prime}}\right|_{L_{\Gamma^{\varepsilon}}^{2}}^{2}
$$

therefore

$$
0 \leq\left(\varepsilon^{\prime}-\varepsilon\right)\left(\left|u^{\varepsilon}\right|_{L_{\Gamma \varepsilon^{\prime}}^{2}}^{2}-\left|u^{\varepsilon^{\prime}}\right|_{L_{\Gamma \varepsilon}^{2}}^{2}\right)
$$

Copyright $@$ by SIAM. Unauthorized reproduction of this article is prohibited. 
and thus $\left|u^{\varepsilon^{\prime}}\right|_{L_{\Gamma^{\varepsilon^{\prime}}}^{2}}^{2} \leq\left|u^{\varepsilon}\right|_{L_{\Gamma^{\varepsilon}}^{2}}^{2}$. Moreover,

$$
\frac{1}{q} \sum_{j=1}^{m}\left(\gamma_{j}^{\varepsilon}\right)^{q}+\frac{\varepsilon}{2}\left|u^{\varepsilon}\right|_{L_{\Gamma^{\varepsilon}}^{2}}^{2} \leq \frac{1}{q} \sum_{j=1}^{m}\left(\gamma_{j}^{*}\right)^{q}+\frac{\varepsilon}{2}\left|u^{*}\right|_{L_{\Gamma^{*}}^{2}}^{2} \leq \frac{1}{q} \sum_{j=1}^{m}\left(\gamma_{j}^{\varepsilon}\right)^{q}+\frac{\varepsilon}{2}\left|u^{*}\right|_{L_{\Gamma^{*}}^{2}}^{2}
$$

thus $\left|u^{\varepsilon}\right|_{L_{\Gamma^{\varepsilon^{\prime}}}^{2}} \leq\left|u^{*}\right|_{L_{\Gamma^{*}}^{2}}$ and (4.2) follows. From (4.2) and (4.4)

$$
\sum_{j=1}^{m}\left(\gamma_{j}^{*}\right)^{q} \leq \sum_{j=1}^{m}\left(\gamma_{j}^{\varepsilon}\right)^{q} \leq \sum_{j=1}^{m}\left(\gamma_{j}^{\varepsilon^{\prime}}\right)^{q} \leq q\left(\frac{1}{q} \sum_{j=1}^{m}\left(\gamma_{j}^{*}\right)^{q}+\frac{\varepsilon^{\prime}}{2}\left|u^{*}\right|_{L_{\Gamma^{*}}^{2}}^{2}\right),
$$

which implies (4.3).

Using the techniques of the proof for Theorem 3.1, we obtain the following optimality condition for $\left(P_{\varepsilon}\right)$.

Proposition 4.3. Let $\left(u^{\varepsilon}, \gamma^{\varepsilon}\right)$ be an optimal solution for $\left(P_{\varepsilon}\right)$ with associated state $x^{\varepsilon}$, and assume that $\gamma_{j}^{\varepsilon}>0, j=1, \ldots, m$, and the regular point condition (3.2) are satisfied. Then there exist $\left(p^{\varepsilon}, \mu^{\varepsilon}\right) \in W^{1, \infty}\left(0, T ; \mathbb{R}^{n}\right) \times \mathbb{R}^{r}$ such that

$$
\left\{\begin{array}{l}
\frac{d}{d t} x^{\varepsilon}=f\left(x^{\varepsilon}, \gamma^{\varepsilon} u^{\varepsilon}\right), \quad x^{\varepsilon}(0)=x_{0}, \quad g\left(x^{\varepsilon}(T)\right)=0 \\
-\frac{d}{d t} p^{\varepsilon}=f_{x}\left(x^{\varepsilon}, \gamma^{\varepsilon} u^{\varepsilon}\right)^{T} p^{\varepsilon}, \quad p^{\varepsilon}(T)=g^{\prime}\left(x^{\varepsilon}(T)\right)^{T} \mu \\
\left(\gamma_{j}^{\varepsilon}\right)^{q-1}+\int_{0}^{T} u_{j}^{\varepsilon}\left(f_{u}\left(x^{\varepsilon}, \gamma^{\varepsilon} u^{\varepsilon}\right)^{T} p^{\varepsilon}\right)_{j} d t=0 \text { for each } j=1, \ldots, m, \\
\left(\varepsilon u^{\varepsilon}+f_{u}\left(x^{\varepsilon}(t), \gamma^{\varepsilon} u^{\varepsilon}(t)\right)^{T} p^{\varepsilon}(t), u-u^{\varepsilon}(t)\right)_{\mathbb{R}^{m}} \geq 0 \text { for a.e. } t \in(0, T), \text { all } u \in U .
\end{array}\right.
$$

The last two equations in (4.5) can be equivalently expressed as

$$
\left\{\begin{array}{l}
\left(\gamma_{j}^{\varepsilon}\right)^{q-1}-\int_{0}^{T}\left|N\left(f_{u}\left(x^{\varepsilon}, \gamma^{\varepsilon} u^{\varepsilon}\right)^{T} p^{\varepsilon}\right)_{j}\right| d t=0, \\
u_{j}^{\varepsilon}=-\operatorname{sgn}_{\varepsilon}\left(f_{u}\left(x^{\varepsilon}, \gamma^{\varepsilon} u^{\varepsilon}\right)^{T} p^{\varepsilon}\right)_{j}, \quad j=1, \ldots, m
\end{array}\right.
$$

where

$$
\operatorname{sgn}_{\varepsilon}(s)=\left\{\begin{array}{cl}
-1 & \text { if } \quad s<-\varepsilon, \\
\frac{s}{\varepsilon} & \text { if } \quad|s| \leq \varepsilon, \\
1 & \text { if } \quad s>\varepsilon,
\end{array} \quad N(s)=\left\{\begin{array}{cll}
|s| & \text { if } & |s| \geq \varepsilon \\
\frac{1}{\varepsilon} s^{2} & \text { if } & |s|<\varepsilon .
\end{array}\right.\right.
$$

5. Semismooth Newton method. In this section we analyze a semismooth Newton method for solving iteratively the optimality system for the regularized problem. It will be convenient to rescale the controls by means of

$$
v_{j}=\gamma_{j} u_{j}, \quad j=1, \ldots, m .
$$

Solving the optimality system (3.18) then amounts to finding a solution to

$$
F(x, p, v, \gamma)=0,
$$

where

$$
F:\left(W^{1,2}\left(0, T ; \mathbb{R}^{n}\right)\right)^{2} \times L^{2}\left(0, T ; \mathbb{R}^{m}\right) \times \mathbb{R}^{m} \rightarrow\left(L^{2}\left(0, T ; \mathbb{R}^{n}\right)\right)^{3} \times \mathbb{R}^{r} \times \mathbb{R}^{m}
$$

Copyright (c) by SIAM. Unauthorized reproduction of this article is prohibited. 
is given by

$$
F(x, p, v, \gamma)=\left(\begin{array}{c}
\frac{d}{d t} x-f(x, v) \\
-\frac{d}{d t} p-f_{x}^{T}(x, v) p \\
v+\gamma \operatorname{sgn}_{\varepsilon}\left(f_{v}(x, v)^{T} p\right) \\
g(x(T)) \\
\left.|\gamma|\right|^{q-2} \gamma-\int_{0}^{T} N\left(f_{v}(x, v)^{T} p\right) d t
\end{array}\right)
$$

Here $\gamma g \operatorname{sgn}_{\varepsilon}$ denotes the vector with coordinates $\gamma_{j}\left(s g n_{\varepsilon}\right)_{j}$, analogously $N$ in the last expression must be interpreted coordinatewise, and the initial condition $x(0)=x_{0}$ is kept as explicit constraint. To solve (5.2) a semismooth Newton method will be used, i.e., we choose an initial condition $\left(x^{0}, p^{0}, v^{0}, \gamma^{0}\right)$ and update $\left(x^{k}, p^{k}, v^{k}, \gamma^{k}\right)$ according to

$$
\left\{\begin{array}{l}
G_{F}\left(x^{k}, p^{k}, v^{k}, \gamma^{k}\right)(\delta x, \delta p, \delta v, \delta \gamma)=-F\left(x^{k}, p^{k}, v^{k}, \gamma^{k}\right) \\
\left(x^{k+1}, p^{k+1}, v^{k+1}, \gamma^{k+1}\right)=\left(x^{k}, p^{k}, v^{k}, \gamma^{k}\right)+(\delta x, \delta p, \delta v, \delta \gamma)
\end{array}\right.
$$

where $G_{F}$ denotes a Newton derivative [IK1]. In the remainder of this section we assume that the control system is linear and we make a special choice for $q$,

$$
\begin{aligned}
& f(x, v)=A x+B v, \quad q=2, \\
& g(x)=G x+d, \text { where } G \in \mathbb{R}^{n \times n} \text { and } d \in \mathbb{R}^{n} .
\end{aligned}
$$

This case contains many of the essential structural ingredients. For $p \in W^{1,2}\left(0, T ; \mathbb{R}^{n}\right)$ we define

$$
\begin{aligned}
& N^{\prime}\left(B^{T} p\right)=\left(n^{\prime}\left(B^{T} p\right)_{1}, \ldots, n^{\prime}\left(B^{T} p\right)_{m}\right)^{T}, \\
& L(t)=e^{A t} B \in \mathbb{R}^{n \times m}, \\
& I_{i}=\left\{t:\left|\left(B^{T} p\right)_{i}(t)\right|<\varepsilon\right\}, \quad i=1, \ldots, m, \\
& \chi_{I}=\left(\chi_{I_{1}}, \ldots, \chi_{I_{m}}\right)^{T},
\end{aligned}
$$

where

$$
n^{\prime}(t)=\left\{\begin{array}{cl}
\operatorname{sgn}(t) & \text { if } \quad t \geq \varepsilon \\
\frac{2}{\varepsilon} t & \text { if } \quad t<\varepsilon
\end{array}\right.
$$

and $\chi_{I_{i}}$ is the characteristic function of the set $I_{i}$. We shall argue in Theorem 5.2 that

$$
G_{F}(x, p, v, \gamma)(\delta x, \delta p, \delta v, \delta \gamma)=\left(\begin{array}{l}
\frac{d}{d t} \delta x-A \delta x-B \delta v \\
-\frac{d}{d t} \delta p-A^{T} \delta p \\
\delta v+\delta \gamma \operatorname{sgn}_{\varepsilon}\left(B^{T} p\right)+\gamma s g n_{\varepsilon}^{\prime}\left(B^{T} p\right) B^{T} \delta p \\
G \delta x(T) \\
\delta \gamma-\int_{0}^{T} N^{\prime}\left(B^{T} p\right) B^{T} \delta p
\end{array}\right)
$$

Copyright (c) by SIAM. Unauthorized reproduction of this article is prohibited. 
provides a Newton derivative at any $(x, p, v, \gamma) \in\left(W^{1,2}\left(0, T ; \mathbb{R}^{n}\right)\right)^{2} \times L^{2}\left(0, T ; \mathbb{R}^{m}\right) \times$ $\mathbb{R}^{m}$ in directions $(\delta x, \delta p, \delta v, \delta \gamma) \in\left(W^{1,2}\left(0, T ; \mathbb{R}^{n}\right)\right)^{2} \times L^{2}\left(0, T ; \mathbb{R}^{m}\right) \times \mathbb{R}^{m}$, where $\delta x(0)=0$.

The following result gives a sufficient condition for well posedness of the Newton iteration (5.3). We require a controllability assumption involving the adjoint state $p^{\varepsilon} \in W^{1,2}\left(0, T ; \mathbb{R}^{n}\right)$ of a solution to $\left(P_{\varepsilon}\right)$. Note that $p^{\varepsilon}(t)=e^{A^{T}(T-t)} p^{\varepsilon}(T)$.

Theorem 5.1 (uniform a priori bound). Consider the case (5.4). Let $p^{\varepsilon} \in$ $W^{1,2}\left(0, T ; \mathbb{R}^{n}\right)$ denote the adjoint state of a solution to $\left(P_{\varepsilon}\right)$, let $\varepsilon \in\left(0, \frac{2}{T}\right)$, and assume that

\section{$\left(A, B \chi_{I\left(p^{\varepsilon}\right)}\right)$ is controllable.}

Then there exist a constant $K_{G}$ and a neighborhood $U\left(p^{\varepsilon}\right)$ of $p^{\varepsilon}$ in $C\left(0, T ; \mathbb{R}^{n}\right)$ such that for every $(x, p, v, \gamma) \in\left(W^{1,2}\left(0, T ; \mathbb{R}^{n}\right)\right)^{2} \times L^{2}\left(0, T ; \mathbb{R}^{m}\right) \times \mathbb{R}^{m}$ with $p \in U\left(p^{\varepsilon}\right)$ the operator $G_{F}(x, p, v, \gamma)$ is invertible and

$$
\left\|G_{F}(x, p, v, \gamma)^{-1}\right\|_{\mathcal{L}\left(\left(L^{2}\right)^{3} \times \mathbb{R}^{n} \times \mathbb{R}^{m},\left(W^{1,2}\right)^{2} \times L^{2} \times \mathbb{R}^{n}\right)} \leq K_{G} .
$$

For the proof of Theorem 5.1 we refer to the appendix. The convergence analysis for the Newton algorithm to solve (5.2) relies on the concept of Newton differentiability, which we briefly recall below. For further discussion we refer to [IK1].

Definition 5.1. The mapping $f: D \subset \mathcal{X} \rightarrow \mathcal{Y}$ is called Newton differentiable at $x \in D$ if there exist an open neighborhood $N(x) \subset D$ and mappings $G: N(x) \rightarrow$ $\mathcal{L}(X, Y)$ such that

$$
\lim _{|h| \rightarrow 0} \frac{|f(x+h)-f(x)-G(x+h) h|_{\mathcal{Y}}}{|h|_{\mathcal{X}}}=0 .
$$

The family $\{G(x): x \in N(x)\}$ is called a Newton map (or Newton derivative) of $f$ at $x$.

To establish superlinear convergence the following result from [HK] will be useful.

Lemma 5.1. Let $f: \mathcal{Y} \rightarrow \mathcal{Z}$ and $g: \mathcal{X} \rightarrow \mathcal{Y}$ be Newton differentiable in open sets $V$ and $U$, respectively, with $U \subset \mathcal{X}, g(U) \subset V \subset \mathcal{Y}$. Assume that $g$ is locally Lipschitz continuous and that there exists a Newton map $G_{f}(\cdot)$ of $f$ which is bounded on $g(U)$. Then the superposition $f \circ g: \mathcal{X} \rightarrow \mathcal{Z}$ is Newton differentiable in $U$ with a Newton map $G_{f} G_{g}$.

TheOREM 5.2 (superlinear convergence). Let $\left(x^{\varepsilon}, p^{\varepsilon}, v^{\varepsilon}, \gamma^{\varepsilon}\right)$ denote a solution to $\left(P_{\varepsilon}\right)$. Under the assumptions of Theorem 5.1 the semismooth Newton iteration (5.3) converges locally superlinearly.

Proof. Since Theorem 5.1 implies that the inverses of $G_{F}(x, p, v, \gamma)$ are uniformly bounded if $p$ is sufficiently close to $p^{\varepsilon}$ in $W^{1,2}\left(0, T ; \mathbb{R}^{n}\right)$, it suffices to argue that $G_{F}$ is in fact a Newton derivative for $F$. The claim then follows from standard results on semismooth Newton algorithms; see, e.g., [IK1, page 268]. To verify Newton differentiability of $F$ it suffices to consider the third and the fifth coordinate of $F$. We recall at first that $\tilde{F}: \varphi \rightarrow \min (0, \varphi)$ is Newton differentiable from $L^{p}\left(0, T ; \mathbb{R}^{n}\right)$ to $L^{q}\left(0, T ; \mathbb{R}^{n}\right)$ if $p>q$ with Newton derivative $G_{\tilde{F}}(\varphi)$ equal -1 , respectively 0 , where $\varphi<0$, respectively $\varphi>0$, coordinatewise, and with arbitrary value for $G_{\tilde{F}}(\varphi)$ where $\varphi=0$. The critical term in the third coordinate of $F$ is given by

$$
p \rightarrow \operatorname{sgn}_{\varepsilon}\left(B^{T} p\right)=\max \left(-1, \min \left(1, \frac{1}{\varepsilon} B^{T} p\right)\right) .
$$

Copyright (C) by SIAM. Unauthorized reproduction of this article is prohibited. 
This mapping is Newton differentiable from $W^{1,2}\left(0, T ; \mathbb{R}^{n}\right)$ to $L^{2}\left(0, T ; \mathbb{R}^{n}\right)$ as a consequence of Lemma 5.1. Similarly

$$
p \rightarrow N\left(B^{T} p\right)=\min \left(-B^{T} p-\frac{1}{\varepsilon}\left(B^{T} p\right)^{2}+\max \left(0,2 B^{T} p\right), 0\right)+\frac{1}{\varepsilon}\left(B^{T} p\right)^{2},
$$

where the square operation acts coordinatewise and is Newton differentiable from $W^{1,2}\left(0, T ; \mathbb{R}^{n}\right)$ to $L^{1}\left(0, T ; \mathbb{R}^{n}\right)$. Consequently $p \rightarrow \int_{0}^{T} N\left(B^{T} p(s)\right) d s$ is Newton differentiable from $W^{1,2}\left(0, T ; \mathbb{R}^{n}\right)$ to $\mathbb{R}$. This concludes the proof.

Remark 5.1. The constraint $\gamma \geq 0$ can be incorporated by means of a complementarity system $\gamma \geq 0, \mu \leq 0, \mu^{T} \gamma=0$, with $\mu \in \mathbb{R}^{m}$. This can equivalently be expressed as $\mu=\min (0, \mu+\gamma)$, where min operates coordinatewise. Since the min operation is known to be Newton-differentiable in $\mathbb{R}^{m}$ (see [IK1, Chapter 8.2]), this equation can be added to the the optimality system (5.2)

6. Numerical results. In this section we describe the implementation of our proposed semismooth Newton method and present numerical results that demonstrate its feasibility.

We use the time discretization of $\left(P_{\varepsilon}\right)$ : Let $\Delta t=\frac{T}{N}$ be a uniform stepsize and

$$
\min \frac{1}{q} \sum_{j=1}^{m}\left(\gamma_{j}\right)^{q}+\frac{\varepsilon}{2} \sum_{j=1}^{m} \sum_{k=1}^{N} \gamma_{j}\left|u_{j}^{k}\right|^{2} \Delta t
$$

subject to

$$
\frac{x^{k}-x^{k-1}}{\Delta t}=f\left(\frac{x^{k}+x^{k-1}}{2}, \gamma u^{k}\right), \quad u^{k} \in U,
$$

and the target constraint $g\left(x^{N}\right)=0$ and $x^{0}=x_{0}$. It is a combination of a second order time integration method (Crank-Nicolson scheme) for the dynamic constraint and a second order quadrature rule (midpoint rule) for the running cost. Let $\left(x^{*}, u^{*}\right)$ be a solution to (6.1)-(6.2). One can derive the necessary optimality condition

$$
\begin{aligned}
& \left(\gamma_{j}\right)^{q-1}-\sum_{k=1}^{N} N\left(\left(f_{u}\left(\frac{x^{k}+x^{k-1}}{2}, \gamma u^{k}\right)^{T} \frac{p^{k}+p^{k-1}}{2}\right)_{j}\right) \Delta t=0, \\
& u_{j}^{k}=-\operatorname{sgn}_{\varepsilon}\left(\left(f_{u}\left(\frac{x^{k}+x^{k-1}}{2}, \gamma u^{k}\right)^{T} \frac{p^{k}+p^{k-1}}{2}\right)_{j}\right), \quad j=1, \ldots, m, \\
& -\frac{p^{k}-p^{k-1}}{\Delta t}=f_{x}\left(\frac{x^{k}+x^{k-1}}{2}, \gamma u^{k}\right)^{T}\left(\frac{p^{k}+p^{k-1}}{2}\right), \quad p^{N}=g^{\prime}\left(x^{N}\right)^{T} \mu .
\end{aligned}
$$

We apply the semismooth Newton method for the system (6.2)-(6.3) for $\left(x^{k}, u^{k}, p^{k}, \gamma\right)$.

The following control system is studied in $[\mathrm{B}, \mathrm{BCB}]$ and is used for our numerical tests. We consider the single-axis slew maneuvers of a simple flexible spacecraft, consisting of a rigid hub and flexible appendages. In state space, with two modes (one rigid and one flexible), we consider the linear system $\dot{x}=A x+B u$ with

$$
A=\left(\begin{array}{cccc}
0 & 1 & 0 & 0 \\
0 & 0 & 0 & 0 \\
0 & 0 & 0 & 1 \\
0 & 0 & -\omega^{2} & 0
\end{array}\right), \quad B=\left(\begin{array}{c}
0 \\
g_{0} \\
0 \\
g_{1}
\end{array}\right)
$$

Copyright $@$ by SIAM. Unauthorized reproduction of this article is prohibited. 
where $\omega$ is the fundamental frequency. We consider rest-to-rest maneuvers. For this case, the initial condition is given by

$$
x_{0}=[-\theta, 0,0,0]^{T},
$$

and the target constraint $x(T)=0$. We use the following values: $\theta=15, \omega=3.0904$, $g_{0}=0.0226$, and $g_{1}=0.00218$. The algorithm is initialized by the solution to the $L^{2}$-minimum norm problem. The $L^{2}$-minimum norm control is unconstrained and its solution is given by

$$
\begin{aligned}
& u(t)=-B^{t} e^{A^{T}(T-t)} \mu, \\
& p(T)=\mu=\left(\int_{0}^{T} e^{A t} B B^{T} e^{A^{T} t} d t\right)^{-1}\left(e^{A T} x_{0}\right) .
\end{aligned}
$$

In the linear case system (6.3) becomes

$$
\left\{\begin{array}{l}
x^{N}=0, \\
\gamma_{j}-\sum_{k=1}^{N} N\left(\left(B^{T} \frac{p^{k}+p^{k-1}}{2}\right)_{j}\right) \Delta t=0, \\
u_{j}^{k}=-\operatorname{sgn}_{\varepsilon}\left(\left(\gamma B^{T} \frac{p^{k}+p^{k-1}}{2}\right)_{j}\right), \quad j=1, \ldots, m, \\
-\frac{p^{k}-p^{k-1}}{\Delta t}-A^{T} \frac{p^{k}+p^{k-1}}{2}=0, \quad p^{N}=\mu, \\
\frac{x^{k}-x^{k-1}}{\Delta t}-A\left(\frac{x^{k}+x^{k-1}}{2}\right)-B\left(\gamma u^{k}\right)=0 .
\end{array}\right.
$$

Thus, one can eliminate $\left(p^{k}, u^{k}, x^{k}\right)$ as a function of $(\gamma, \mu)$, i.e., $p^{k}$ is determined by solving the adjoint equation backward, knowing $\mu, u^{k}$ is determined by the optimality condition, knowing $\gamma$, and then the state $x^{k}$ is determined by the state equation. In this way system (6.5) can equivalently written as the following reduced equation for $(\gamma, \mu)$ :

$$
F(\gamma, \mu)=\left(\begin{array}{l}
x^{N} \\
\gamma_{j}-\sum_{k=1}^{N} N\left(B^{T} \frac{p^{k+1}+p^{k}}{2}\right)_{j} \Delta t
\end{array}\right)=0 .
$$

Here, the Newton derivative of $F$ is

$$
F^{\prime}(\gamma, \mu)(\delta \gamma, \delta \mu)=\left(\begin{array}{l}
\delta x^{N} \\
\delta \gamma_{j}-\sum_{k=1}^{N} N^{\prime}\left(B^{T} \frac{p^{k}+p^{k-1}}{2}\right) B^{T}\left(\frac{p^{k}+p^{k-1}}{2}\right) \Delta t
\end{array}\right),
$$

where

$$
\begin{aligned}
& -\frac{\delta p^{k}-\delta p^{k-1}}{\Delta t}-A^{T} \frac{p^{k}+p^{k-1}}{2}=0, \quad \delta p^{N}=\delta \mu \\
& \delta u_{j}^{k}=-s g n_{\varepsilon}^{\prime}\left(\left(\gamma B^{T} \frac{p^{k}+p^{k-1}}{2}\right)_{j}\right)\left(\delta \gamma B^{T} \frac{p^{k}+p^{k-1}}{2}+\gamma B^{T} \frac{p^{k}+p^{k-1}}{2}\right)_{j} \\
& \frac{\delta x^{k}-\delta x^{k-1}}{\Delta t}-A\left(\frac{\delta x^{k}+\delta x^{k-1}}{2}\right)-B\left(\delta \gamma u^{k}+\gamma \delta u^{k}\right)=0
\end{aligned}
$$

Copyright $@$ by SIAM. Unauthorized reproduction of this article is prohibited. 

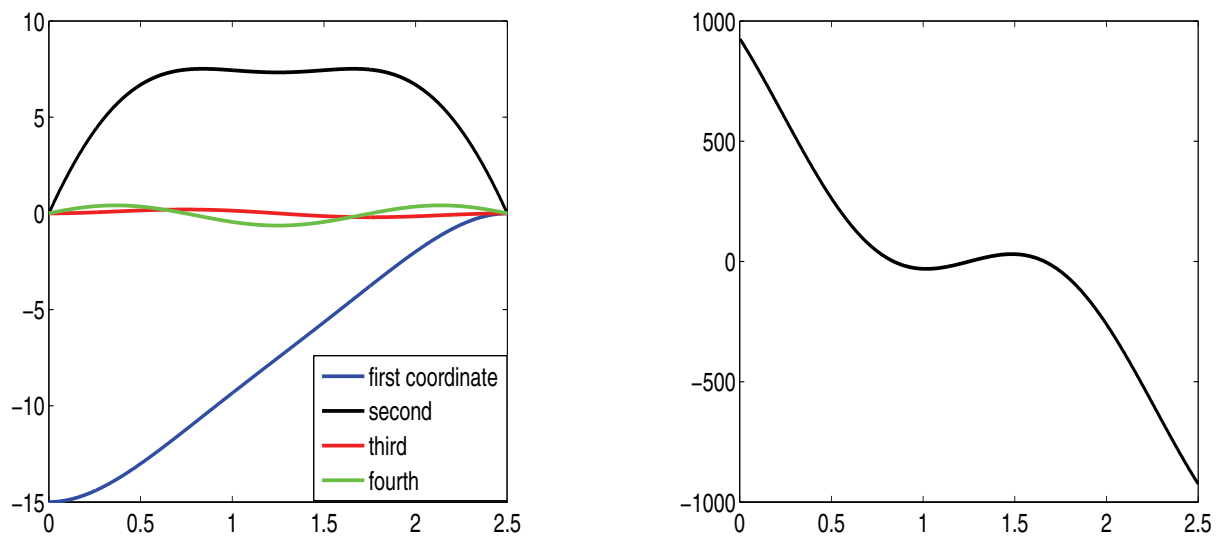

FIG. 1. The trajectory and control of the $L^{2}$-minimum norm solution for the control of spacecraft with the fixed time horizon $T=2.5$.

TABLE 1

Numerical solution of minimum effort $\gamma$ for the control of a spacecraft with different time horizon $T$.

\begin{tabular}{|r|r|c|}
\hline$T$ & Minimum $\gamma$ & Number of switches \\
\hline 2 & 1372.1 & 3 \\
\hline 2.5 & 590.13 & 3 \\
\hline 3 & 331.73 & 3 \\
\hline 10 & 27.097 & 3 \\
\hline
\end{tabular}

The proposed algorithm can now be summarized.

Algorithm.

- Initialize $\mu$ and $\gamma=\max \left(\left|u^{k}\right|\right)$ by (6.4).

- Compute $\left(p^{k}, u^{k}, x^{k}\right)$ by the last three equations of (6.5).

- Solve $F(\gamma, \mu)(\delta \gamma, \delta \mu)+F(\gamma, \mu)=0$ with (6.6).

- Update $\left(\begin{array}{l}\gamma^{+} \\ \mu^{+}\end{array}\right)=\left(\begin{array}{l}\gamma \\ \mu\end{array}\right)+\alpha\left(\begin{array}{l}\delta \gamma \\ \delta \mu\end{array}\right)$.

The step-size $\alpha=.25$ is chosen up to 10 iterates; otherwise $\alpha=1$.

For a fixed time horizon $T=2.5$, Figure 1 shows the resulting trajectory and control. The resulting minimum effort $\gamma$ for different horizons $T$ is shown in Table 1 . There are three switches in the control. Figure 2 shows the resulting trajectory and control for the minimum effort problem.

In order to investigate the multiple control input case we include another control input which can control the first and third coordinates. The problem can be stated as

$$
\frac{d x(t)}{d t}=A x(t)+u_{1}(t) \gamma_{1} B_{1} u_{1}(t)+\gamma_{2} B_{2} u_{2}(t)
$$

with

$$
B_{1}=\left[0, g_{0}, 0, g_{1}\right]^{T}, \quad B_{2}=\left[g_{2}, 0, g_{3}, 0\right]^{T} \text {. }
$$

Copyright $@$ by SIAM. Unauthorized reproduction of this article is prohibited. 

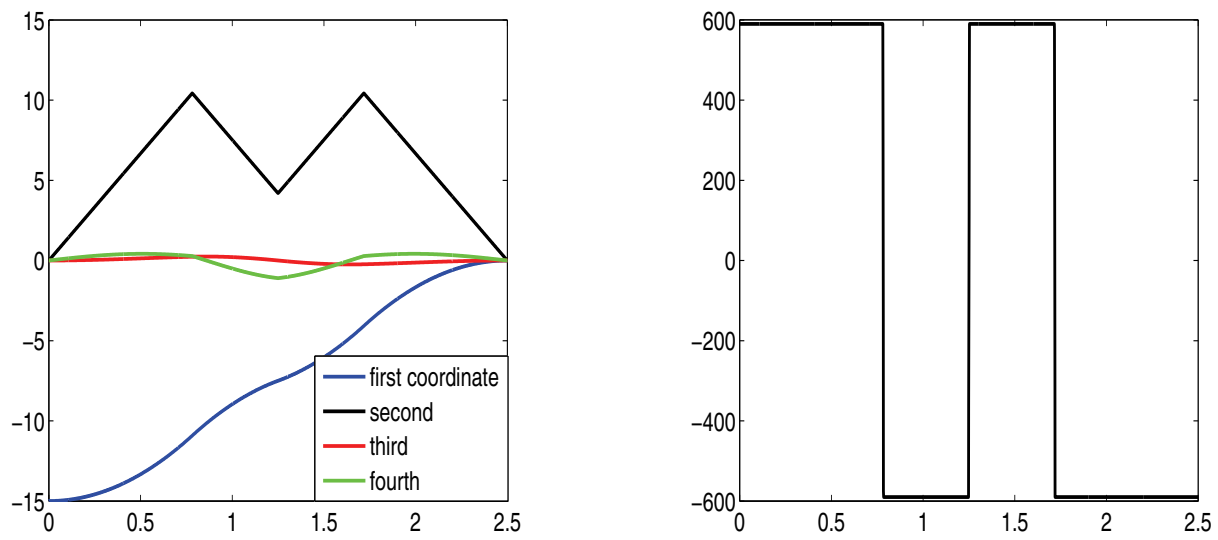

FIG. 2. The trajectory and control of minimum effort problem for the control of spacecraft, the minimum effort $\gamma=590.1336$.
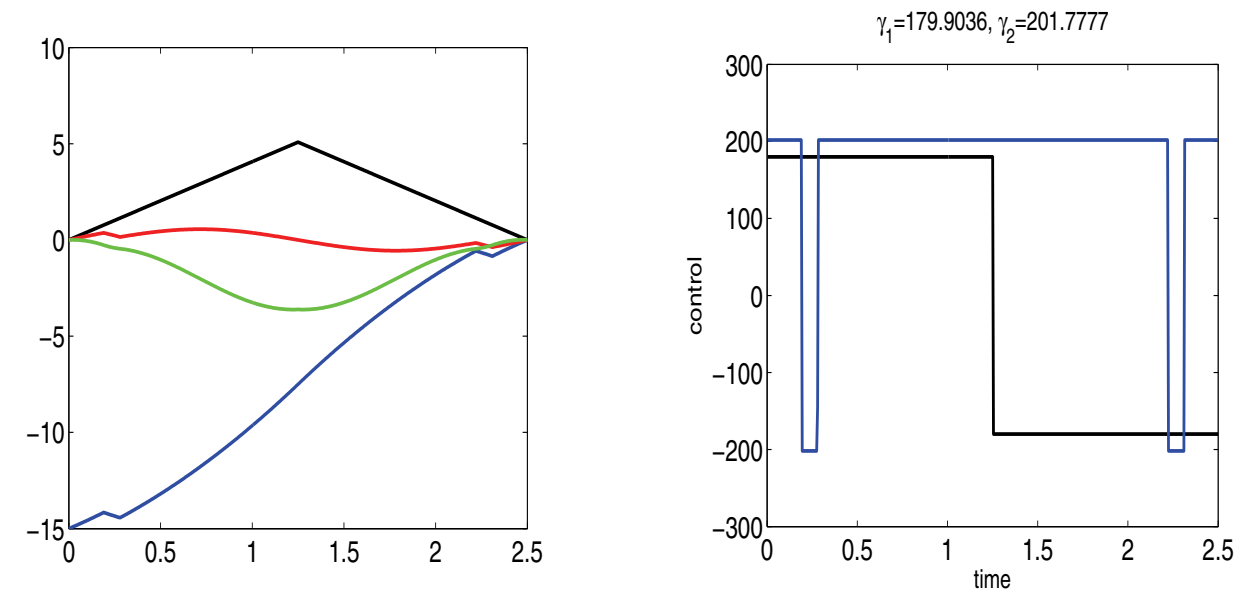

FIG. 3. The trajectory and control of minimum effort problem for the control of spacecraft with two control inputs.

Here we set $g_{2}=0.02, g_{3}=0.01$. With the constrained control $\left|u_{1}(t)\right| \leq 1,\left|u_{2}(t)\right| \leq 1$, we consider the control problem of minimizing the cost functional

$$
\min \frac{1}{2}\left(\gamma_{1}^{2}+\gamma_{2}^{2}\right)
$$

For fixed time horizon $T=2.5$, the minimum control effort is given by

$$
\gamma_{\mathrm{opt}}=\left(\begin{array}{l}
179.91 \\
201.78
\end{array}\right)
$$

Figure 3 shows that the control $u_{1}$ has one switch while control $u_{2}$ has four switches.

Copyright $@$ by SIAM. Unauthorized reproduction of this article is prohibited. 
A. Appendix. In this appendix we provide the proof of Theorem 5.1.

Proof. For arbitrary $w=\left(w_{1}, \ldots, w_{5}\right)^{T} \in\left(L^{2}\left(0, T ; \mathbb{R}^{n}\right)\right)^{3} \times \mathbb{R}^{n} \times \mathbb{R}^{m}$, we need to consider

$$
\left\{\begin{array}{l}
\frac{d}{d t} \delta x-A \delta x-B \delta v=w_{1}, \quad \delta x(0)=0 \\
-\frac{d}{d t} \delta p-A^{T} \delta p=w_{2} \\
\delta v+\delta \gamma \operatorname{sgn}_{\varepsilon}\left(B^{T} p\right)+\gamma s g n_{\varepsilon}^{\prime}\left(B^{T} p\right) B^{T} \delta p=w_{3} \\
G \delta x(T)=w_{4} \\
\delta \gamma-\int_{0}^{T} N^{\prime}\left(B^{T} p\right) B^{T} \delta p d t=w_{5} .
\end{array}\right.
$$

We define

$$
\begin{aligned}
& \hat{w}_{1}=\int_{0}^{T} e^{A(T-s)} w_{1}(s) d s \\
& \hat{w}_{2}=-\gamma \int_{0}^{T} L(T-s) s g n_{\varepsilon}^{\prime}\left(B^{T} p(s)\right) \int_{s}^{T} L^{T}(\sigma-s) w_{2}(\sigma) d \sigma d s, \\
& \hat{w}_{3}=\int_{0}^{T} L(T-s) w_{3}(s) d s \\
& \tilde{w}_{2}=\int_{0}^{T} N^{\prime}\left(B^{T} p(s)\right) \int_{t}^{T} L^{T}(s-t) w_{2}(s) d s d t .
\end{aligned}
$$

From the first two equations in (A.1) we have

$$
\left\{\begin{array}{l}
\delta x(t)=\int_{0}^{t} L(t-s) \delta v d s+\int_{0}^{t} e^{A(t-s)} w_{1}(s) d s, \\
\delta p(t)=e^{A^{T}(T-t)} \delta p(T)+\int_{t}^{T} e^{A^{T}(s-t)} w_{2}(s) d s .
\end{array}\right.
$$

From the fourth equation in (A.1) we find

$$
\begin{aligned}
& w_{4}=G \delta x(T) \\
& =G\left[\hat{w}_{1}+\hat{w}_{3}-\gamma \int_{0}^{T} L(T-s) \operatorname{sgn}_{\varepsilon}^{\prime}\left(B^{T} p\right) B^{T} \delta p d s-\int_{0}^{T} L(T-s) \delta \gamma \operatorname{sgn}_{\varepsilon}\left(B^{T} p\right) d s\right] \\
& =G\left[\hat{w}_{1}+\hat{w}_{3}-\gamma \int_{0}^{T} L(T-s) \operatorname{sgn}_{\varepsilon}^{\prime}\left(B^{T} p\right) L^{T}(T-s) \delta p(T) d s+\hat{w}_{2}\right. \\
& \left.\quad-\int_{0}^{T} L(T-s) \delta \gamma \operatorname{sgn}_{\varepsilon}\left(B^{T} p\right) d s\right]
\end{aligned}
$$

and hence

$$
\begin{aligned}
& \frac{\gamma}{\varepsilon} \int_{0}^{T} L(T-s) \chi_{I} L^{T}(T-s) \delta p(T) d s+\int_{0}^{T} L(T-s) \delta \gamma \operatorname{sgn}_{\varepsilon}\left(B^{T} p\right) d s \\
& =\hat{w}_{1}+\hat{w}_{2}+\hat{w}_{3}-G^{-1} w_{4}=: r_{1} .
\end{aligned}
$$

Similarly we obtain

$$
\delta \gamma-\int_{0}^{T} N^{\prime} L^{T}(T-t) \delta p(T) d t-\int_{0}^{T} N^{\prime} \int_{t}^{T} L^{T}(s-t) w_{2}(s) d s d t=-w_{5},
$$

Copyright (c) by SIAM. Unauthorized reproduction of this article is prohibited. 
and hence

$$
\delta \gamma-\int_{0}^{T} N^{\prime} L^{T}(T-t) \delta p(T) d t=\int_{0}^{T} N^{\prime} \int_{t}^{T} L^{T}(s-t) w_{2}(s) d s d t-w_{5},
$$

which implies that

$$
\delta \gamma-\int_{0}^{T} N^{\prime} L^{T}(T-t) \delta p(T) d t=\tilde{w}_{2}-w_{5}=: r_{2},
$$

where $N^{\prime}=N^{\prime}\left(B^{T} p\right)$. Combining (A.3) and (A.4) we arrive at

$$
M(p)\left(\begin{array}{c}
\delta p(T) \\
\delta \gamma
\end{array}\right)=\left(\begin{array}{c}
r_{1} \\
r_{2}
\end{array}\right)
$$

where

$$
M(p)=\left(\begin{array}{cc}
\frac{1}{\varepsilon} \int_{0}^{T} L(T-s) \chi_{I} L^{T}(T-s) d s & \int_{0}^{T} L(T-s)(\cdot) \operatorname{sgn}_{\varepsilon}\left(B^{T} p\right) d s \\
-\int_{0}^{T} N^{\prime}\left(B^{T} p\right) L^{T}(T-s) d s & I
\end{array}\right)
$$

For $(\delta p(T), \delta \gamma) \in \mathbb{R}^{n+m}$ we find by (A.6) that

$$
\begin{aligned}
& (M(p)(\delta p(T), \delta \gamma),(\delta p(T), \delta \gamma))_{\mathbb{R}^{n+m}} \\
& \quad=\frac{1}{\varepsilon} \int_{0}^{T}\left|\chi_{I} L^{T}(T-s) \delta p(T)\right|_{\mathbb{R}^{n}}^{2} d s+\int_{0}^{T} \delta p^{T} L(T-s) \delta \gamma s g n_{\varepsilon}\left(B^{T} p\right) d s \\
& \quad-\int_{0}^{T} \delta \gamma^{T} N^{\prime} L^{T}(T-s) \delta p(T) d s+|\delta \gamma|_{\mathbb{R}^{m}}^{2} \\
& \quad=\frac{1}{\varepsilon} \int_{0}^{T}\left|\chi_{I} L^{T}(T-s) \delta p(T) d s\right|_{\mathbb{R}^{n}}^{2}+\int_{0}^{T} \delta p^{T}\left(L(T-s)\left(s g n_{\varepsilon}\left(B^{T} p\right)-N^{\prime}\right) \delta \gamma d s\right)+|\delta \gamma|_{\mathbb{R}^{m}}^{2} \\
& \quad=\frac{1}{\varepsilon} \int_{0}^{T}\left|\chi_{I} L^{T}(T-s) \delta p(T) d s\right|_{\mathbb{R}^{n}}^{2}-\int_{0}^{T} \delta p^{T}\left(L(T-s) \operatorname{sgn} n_{\varepsilon}\left(B^{T} p\right) \chi_{I} \delta \gamma d s\right)+|\delta \gamma|_{\mathbb{R}^{m}}^{2} \\
& \quad \geq \frac{1}{\varepsilon} \int_{0}^{T}\left|\chi_{I} L^{T}(T-s) \delta p(T) d s\right|_{\mathbb{R}^{n}}^{2}-\frac{\varepsilon}{2} \int_{0}^{T}\left|s g n_{\varepsilon}\left(B^{T} p\right) \delta \gamma \chi_{I}\right|_{\mathbb{R}^{m}}^{2} d s+|\delta \gamma|_{\mathbb{R}^{m}}^{2} \\
& \quad \geq \frac{1}{\varepsilon} \int_{0}^{T}\left|\chi_{I} L^{T}(T-s) \delta p(T) d s\right|_{\mathbb{R}^{n}}^{2}+\left(1-\frac{\varepsilon T}{2}\right)|\delta \gamma|_{\mathbb{R}^{m}}^{2} .
\end{aligned}
$$

For $p^{\varepsilon}$ and $\eta<\varepsilon<\frac{2}{T}$ define

$I_{i}^{\eta}\left(p^{\varepsilon}\right)=\left\{t:\left|\left(B^{T} p^{\varepsilon}\right)_{i}(t)\right|<\varepsilon-\eta\right\}, \quad i=1, \ldots, m, \quad \chi_{I^{\eta}\left(p^{\varepsilon}\right)}=\left(\chi_{I_{1}^{\eta}\left(p^{\varepsilon}\right)}, \ldots, \chi_{I_{m}^{\eta}\left(p^{\varepsilon}\right)}\right)^{T}$,

and note that $I_{i}^{0}\left(p^{\varepsilon}\right)=I_{i}\left(p^{\varepsilon}\right)$. Since, by assumption, $\left(A, B \chi_{I\left(p^{\varepsilon}\right)}\right)$ is controllable, the matrix $\int_{0}^{T} L(T-s) \chi_{I\left(p^{\varepsilon}\right)} L^{T}(T-s) d s$ is positive definite in $\mathbb{R}^{n}$; see, e.g., [HL]. Since $p^{\varepsilon}=e^{A^{T}(T-t)} p(T)$, there exists $\eta \in(0, \varepsilon)$ such that $\int_{0}^{T} L(T-s) \chi_{I^{\eta}\left(p^{\varepsilon}\right)} L^{T}(T-s) d s$ is positive definite.

Let $U\left(p^{\varepsilon}(T)\right)$ be a neighborhood in $\mathbb{R}^{n}$ such that for all $p(T) \in U\left(p^{\varepsilon}(T)\right)$ we have

$$
\left|B^{T} p(\cdot)-B^{T} p^{\varepsilon}(\cdot)\right|_{L^{\infty}\left(0, T ; \mathbb{R}^{m}\right)} \leq \eta .
$$

Copyright (c) by SIAM. Unauthorized reproduction of this article is prohibited. 
Then $I_{j}^{\eta}\left(p^{\varepsilon}\right) \subset I_{j}(p)$ for all $j=1, \ldots, m$. As a consequence $\left(A, B \chi_{(p)}\right)$ is controllable for all $p \in U\left(p^{\varepsilon}\right), M(p)$ is invertible, and

$$
\left\|M(p)^{-1}\right\|_{\mathbb{R}^{(n+m) \times(n+m)}} \leq K_{M}
$$

for a constant $K_{M}$ independent of $p \in U\left(p^{*}\right)$. Estimate (5.6) follows from the definition of $r_{1}, r_{2}$, for $\delta p(T), \delta \gamma$, from (A.2) for $\delta p$, from (A.1) for $\delta v$, and again from (A.2) for $\delta x$.

\section{REFERENCES}

[BCB] J. Z. Ben-Asher, E. M. Cliff, And J. A. Burns, Computational methods for the minimum effort problem with applications to spacecraft rotational maneuvers, in Proceedings of the 1989 IEEE International Conference on Control and Applications, 1989, pp. 1-7.

[B] J. A. Breakwell, Optimal feedback slewing of flexible spacecraft, J. Guidance Control, 4 (1981), pp. 472-479.

[BMT] F. Bonnans, P. Martinon, And E. TrÉlat, Singular arcs in the generalized Goddard's problem, J. Optim. Theory Appl., 139 (2008), pp. 439-461.

[BPW] Ch. Büskens, H. J. Pesch, and S. Winderl, Real-time solutions of bang-bang and singular optimal control problems, in Online Optimization of Large Scale Systems, M. Grötschel et al., eds., Springer-Verlag, Berlin, 2001, pp. 129-142.

[HK] M. Hintermüller And K. KUnisch, PDE-constrained optimization subject to pointwise constraints on the control, the state, and its derivative, SIAM J. Optim., 20 (2009), pp. 1133-1156.

[HL] H. Hermes and J. LaSAlle, Functional Analysis and Time Optimal Control, Academic Press, New York, London, 1969.

[IK1] K. Ito AND K. Kunisch, On the Lagrange Multiplier Approach to Variational Problems and Applications, SIAM, Philadelphia, 2008.

[IK2] K. ITO AND K. KUnISCH, The primal-dual active set method for nonlinear optimal control problems with bilateral constraints, SIAM J. Control Optim., 43 (2004), pp. 357-376.

[IK3] K. ITO AND K. Kunisch, Semismooth Newton methods for time-optimal control for a class of ODEs, SIAM J. Control Optim., 48 (2010), pp. 3997-4013.

[KN] C. Y. KAYA AND J. L. NOAKES, Computational methods for time-optimal switching controls, J. Optim. Theory Appl., 117 (2003), pp. 69-92.

[MS] J. W. Macki AND A. Strauss, Introduction to Optimal Control Theory, Springer-Verlag, Berlin, 1982.

[MAG] H. Maurer, I. Altrogge, And N. Goris, Optimization methods for solving bang-bang control problems with state constraints and the verification of sufficient conditions, in Proceedings of the 44th IEEE Conference on Decision and Control, and the European Control Conference, 2005, Seville, Spain, 2005.

[MBKK] H. Maurer, C. Büeskens, J.-H. R. Kim, and C. Y. Kaya, Optimization methods for the verification of second order sufficient conditions for bang-bang controls, Optimal Control Appl. Methods, 26 (2005), pp. 129-156.

[MZ] H. MAURER AND J. Zowe, First and second order necessary and sufficient optimality conditions for infinite-dimensional programming problems, Math. Programming, 16 (1979), pp. 98-110.

[MB] E.-B. Meier AND A. E. BRYson, JR., Efficient algorithms for time-optimal control of a two-link manipulator, J. Guidance Control Dynam., 13 (1990), pp. 859-866.

[N] L. W. Neustadt, Minimum effort control systems, J. SIAM Control Ser. A, 1 (1962), pp. $16-31$.

[U] M. Ulbrich, Semismooth Newton methods for operator equations in function spaces, SIAM J. Optim., 13 (2002), pp. 805-842.

[VM] G. Vossen And H. MAUrer, On L1-minimization in optimal control and applications to robotics, Optimal Control Appl. Methods, 27 (2006), pp. 301-321.

Copyright $@$ by SIAM. Unauthorized reproduction of this article is prohibited. 